\title{
A Highly Efficient Protocol To Establish Regeneration And Genetic Transformation Systems And Create Targeted Mutations Using CRISPR/Cas9 in Lycium Ruthenicum
}

\author{
Wang Wang \\ Beijing Forestry University \\ Hai Wang \\ Beijing Forestry University \\ Jiangmiao Liu \\ Beijing Forestry University \\ Tong Li \\ Beijing Forestry University \\ Huien Zhao ( $\square$ zhaohuien@bjfu.edu.cn ) \\ https://orcid.org/0000-0002-9343-7249
}

\section{Research}

Keywords: Black wolfberry, CRISPR/Cas9, gene editing, fw2.2

Posted Date: April 8th, 2021

DOI: https://doi.org/10.21203/rs.3.rs-398418/v1

License: (c) (i) This work is licensed under a Creative Commons Attribution 4.0 International License.

Read Full License 


\section{Abstract}

Background: The CRISPR/Cas9 system has rapidly become the preferred tool for various biological sequencing projects due to its high efficiency, specificity, simplicity and versatility, and it has been utilized for targeted genomic alternations in several important plants of Solanaceae, including tomato, tobacco, potato, petunia and groundcherry. Wolfberry is the sixth most important solanaceous crop in China following potato, tomato, eggplant, pepper and tobacco. To date, there has been no report on CRISPR/Cas9 technology to improve Lycium ruthenicum due to the unknown genome and the lack of efficient regeneration and genetic transformation systems.

Results: In this study, we established a suitable regeneration and genetic transformation system of Lycium ruthenicum, the fw2.2 gene was identified, which was the first fruit weight gene identifified from tomato and accounted for $30 \%$ of the variation in fruit size. The gene editing of black wolfberry were carried out by CRISPR/ Cas9 for the first time here with a very high editing efficiency (95.45\%) in $f w 2.2$. Four homozygous mutations and nine biallelic mutations were obtained from T0 generation plants.

Conclusions: These results suggest that the CRISPR/Cas9 system is effective for gene editing study of black wolfberry, and we expect this approach to be routinely applied to this important economic fruit.

\section{Background}

Wolfberry is used as a traditional medicine in China and other Asian countries owing to the abundance of anthocyanins, trace minerals, vitamins and polysaccharides in the fruit[1,2]. There are about 160000 $\mathrm{hm}^{2}$ of production area and 410000 tonnes of yield with 18.7 billion RMB value in China in 2018. It was thought Black Chinese wolfberry has more important value than other fructus lycii with procyanidine-rich content, as a result, the artificial cultivation of Lycium ruthenicum has been greatly developed in North West China. The cultivation of black wolfberry is also labor-intensive industry and the fruit is mostly hand harvested. Small fruit and dense sharp thorn make it more difficult for harvest. The large fruit size is an important fruit quality characteristic which affects its market competitiveness and nutritional value. As a producer, obtaining new large-fruited cultivars is a major breeding goal for grower profifitability. Therefore, understanding the genetic background of fruit size-related genes will significantly improve the breeding efficiency of large-fruit varieties, which will also greatly promote the use of small-fruit wild germplasm, as it will reduce the number of generations required to obtain the commercial fruit size needed for a new cultivar. The regulation of fruit size by genetic changes is the most widely studied in tomato to date (Solanum lycopersicum L.) [3, 4]. fw2.2 was the first fruit weight gene identifified from tomato as a result of domestication, it is a major quantitative trait locus that regulates fruit size and weight in tomato, and natural genetic variation at this locus alone can change the size of fruit by up to $30 \%$ in tomato between large, domesticated tomatoes (Lycopersicon esculentum Mill.) and their small-fruited wild relatives [5].The "small-fruit" alleles at the fw2.2 locus exists in all wild tomatoes, whereas all cultivated tomatoes tested are fixed for "large-fruit" alleles [6]. The higher fw2.2 transcript levels negatively regulate cell division, which result in a decrease in cell number, therefore affect the mitotic activity during early fruit 
development $[5,7]$. Many studies have demonstrate that cell number is a key factor determining fruit size in fruit crops including peach [8], sweet cherry[9], olive[10], and apple[11]. Due to the critical roles of fw2.2 in regulating cell number, orthologs to these $f w 2.2$ genes for fruit sizes are further studied[12, 13]. Therefore, fw2.2 was selected for establishing the technology of gene editing in Lycium here.

The CRISPR/Cas9 system has been utilized for targeted genomic alternations in several important plants of Solanaceae, including tobacco, tomato, potato, petunia and groundcherry $[14,15]$. Among horticultural crops, tomato has received much more attention regarding genome editing than other crops: $\sim 42 \%$ of genome-editing studies have involved tomato, whereas $~ 13 \%$ have involved potato[15]. CRISPR/Cas9mediated knock-out of polyphenol oxidase genes in eggplant has also been reported[16]. However, this powerful tool for genome editing has not yet been used in Lycium.

In this study, we established an efficient regeneration and genetic transformation system for Lycium ruthenicum, and we first adapted a schematic workflow and cloning strategy for black wolfberry CRISPR/Cas9 system in order to breeding elite cultivars with bigger fruits in facing on the harvest difficulty since the stems are full of thorn. The results show that this CRISPR/Cas9 system is effective in Lycium ruthenicum.

\section{Methods}

\section{Plant materials}

Wild Lycium ruthenicum seeds were obtained from Alxa, Inner Mongolia, and rinsed under running water for 2 hours, then $75 \%$ alcohol was used to disinfect for 30 s, rinsed with sterile water once, then rinsed with $4 \%$ sodium hypochlorite for 3 minutes, the seeds were rinsed with sterile water 3 times and then inoculated in $1 / 2 \mathrm{MS}$ medium, placed at $25^{\circ} \mathrm{C}$ and $14 \mathrm{~h} / \mathrm{d}$ light (1500 2000lx) in the tissue culture room to obtain tissue culture seedlings for later gene editing study.

\section{fw2.2gene cloning and sequence analysis}

The fw2.2 sequence of Solanum lycopersicum (NM_001321132.1), Lycopersicon pennellii (AY097189.1), Physalis coztomatl (KJ155739.1), Physalis lanceifolia (KJ155740.1), Physalis pruinosa (KJ155742.1), Physalis peruviana (KJ155745.1), Physalis ixocarpa (KJ155746.1), Physalis mexicana (KJ155748.1) were used as seed sequences to search the Lycium ruthenicum transcriptome sequencing database (SRA:SSR7700825), and a highly homologous sequence was obtained based on BLAST search. The Pfam database (http://pfam.janelia.org/)[30] and the Conserved Domain Database (CDD) of the NCBI[31] were used to analyze the obtained sequence that met the known conserved domains and motifs requirements. The RNA and genomic DNA were extracted from Lycium ruthenicum using Tiangen Kit, and the gene-specific primers were designed to amplify the fw2.2 sequence (Additional file 1 Table S4). The exons and intron regions were analyzed after the full length of the fw2.2 gene was got. The sequences of fw2.2 proteins producing significant alignments were searched on BLASTP programs (https://blast.ncbi.nlm.nih.gov/Blast.cgi ) to construct phylogenetic tree, and the neighbor-joining (NJ) 
method of the MEGA-X was used to analyze phylogenetic relationships, the confidence limits of each branch in the phylogenetic tree were assessed by 1000 bootstrap replications and expressed as percentage values.

\section{CRISPR/Cas9 vector construction}

The CRISPR online design tool (http://crispr.dbcls.jp/ ) was used to analyze the target location and GC content, etc., and the tomato (Solanum lycopersicum) genome SL2.40 was used as a reference to assess the target specificity (Additional file 1 Table S2). The single target vector construction method was shown in Fig. 4. A single target editing primers fw2.2-1-sgRNA and fw2.2-2-sgRNA with target adapter were designed (Additional file 1 Table S5), then the primers were used to anneal and pair following the procedure $(20 \mu \mathrm{l}$ reaction system contains $1 \mu \mathrm{l}$ forward primer, $1 \mu \mathrm{l}$ reverse primer and $18 \mu \mathrm{l}$ anneal buffer, under the condition of $95^{\circ} \mathrm{C} 1 \mathrm{~min} \otimes 0.1^{\circ} \mathrm{C} / \mathrm{s}$ cooling down to $25^{\circ} \mathrm{C}$ ). The pCAMBIA1300-sgRNA/Cas 9 vector was digested with Bsal (NEB) under $37^{\circ} \mathrm{C}$ for $2 \mathrm{~h}$ ( $25 \mu$ reaction system contains $2 \mu \mathrm{g}$ plasmid vector, $0.5 \mu \mathrm{l} \mathrm{Bsal,} 2.5 \mu \mathrm{l} 10 \times$ Cutsmart Buffer and ddH2O). The double-stranded primer was then ligated with the digested vector to obtain single-target editing vector pCAMBIA1300-fw2.2-1 and pCAMBIA1300fw2.2-2.

The dual target vector construction method was shown in Fig. 5. The above-obtained pCAMBIA1300fw2.2-1 and pCAMBIA1300-fw2.2-2 were used as templates, the primers containing EcoRI restriction site were used for PCR amplification to obtain fw2.2-AtU6-sgRNA1 and fw2.2-AtU6 -sgRNA2 (Additional file 1 Table S6). The two sgRNA expression cassettes were respectively digested with EcoRI and then ligated with T4 ligase to obtain the two-site expression cassette fw2.2-AtU6-sgRNA1-AtU6-sgRNA2. Both the fw2.2-AtU6-sgRNA1-AtU6-sgRNA2 and pCAMBIA1300-sgRNA/Cas9 vector were digested with HindllI and Xmal, respectively, and then ligated with T4 ligase to obtain pCAMBIA1300-fw2. 2-1/2 dual-site expression vector.

\section{Optimization of regeneration system and Agrobacterium-mediated transformation of Lycium ruthenicum}

The leaves of the Lycium ruthenicum tissue culture seedlings were cut into about $0.5 \mathrm{~cm}$ and then transferred to MS medium (A1-A12) supplemented with various concentrations and combinations of 6BA, NAA and 2, 4-D to induce callus (Additional file 1 Table S1). The callus induction rate was counted after 30 days based on three experiments using 30-40 explants in each treatment. The callus was subsequently transferred to MS medium (B1-B10) supplemented with various concentrations of 6-BA and NAA to induce differentiation (Additional file 1 Table S1). 30-40 explants were used in each treatment of three experiments, and explants were subcultured at intervals of 20 days. The differentiation rate and multiplication coefficient were counted and recorded after 60 days. The optimal regeneration system of Lycium ruthenicum was determined based on the above callus induction and differentiation experiments.

The leaves of Lycium ruthenicum tissue culture seedlings were used for Agrobacterium transformation. The influence of hygromycin concentration, infection time, co-cultivation time, bacterial solution concentration and acetosyringone concentration were comprehensively analyzed to explore the optimal 
genetic transformation conditions (Additional file 1 Table S3). The Agrobacterium cells were collected and resuspended in MS liquid medium after expanded cultivation in LB medium containing $50 \mathrm{mg} / \mathrm{L} \mathrm{Kan}$, $50 \mathrm{mg} / \mathrm{L}$ Gen and 50mg/L Rif. After infecting in a sterile test tube containing Agrobacterium suspension, the leaves of Lycium ruthenicum were transferred to co-culture medium (MS+0.5mg/L6-

$\mathrm{BA}+0.5 \mathrm{mg} / \mathrm{LNAA}$ ) for two days in the dark at $25^{\circ} \mathrm{C}$, and then transferred to resistant callus selection medium (MS+ 0.5mg/L6-BA+0.5mg/LNAA+40mg/L Hyg+200 mg/L Carb). After that, the resistant callus were transferred to the medium (MS+0.2mg/L6- BA+0.05mg/LNAA+40mg/L Hyg+200 mg/L Carb) to obtain resistant buds. With 2 rounds of selection and culture, the resistant buds were cut and transferred to rooting medium (1/2 MS+200 mg/L Carb) to obtain the gene editing tissue culture seedlings of Lycium ruthenicum.

\section{Identification of geneedited Lycium ruthenicum}

The unedited tissue culture seedling was used as a control, the genomic DNA was extracted as a template for amplification and sequencing. The detection primers were designed in the upstream and downstream of the gene editing site (Additional file 1 Table S7). The mutation type can be observed from the sequencing chromatograms (Fig. 6 and Additional file 2), when the target sequence has a heterozygous mutation (only one chromosome is mutated, while the other is not) or biallelic mutations (different mutations on two chromosomes), there will be overlapping peaks after the target site. When the target sequence has a homozygous mutation (two chromosomes have the same mutation) or no mutation, a single peak appears. The mutation sequences can be read directly from the sequencing files. Based on this, the mutation types were counted, and the editing efficiency were also counted following the formula: gene editing efficiency $(\%)=$ (number of gene-edited seedlings / number of resistant seedlings ) $\times 100 \%$.

\section{Quantitative real-time PCR (qRT-PCR) analysis of fw2.2 in geneedited seedlings}

In order to study whether gene editing has an effect on the expression of fw2.2, the wild Lycium ruthenicum seedlings and the gene-edited seedlings including X9 (homozygous), X17 (heterozygous), X20 (biallelic) and X21 (heterozygous) were selected for quantitative real-time PCR analysis. Actin (HQ415754.1) was used as an internal control. The primers used for qRT-PCR were designed on NCBI( https://www.ncbi.nlm.nih.gov/tools/primer-blast/) including actin -F: TACGAGGGTTACGCTTTGCC, actin R: TTCCCGTTCAGCAGTGGTTG, Fw2.2-F: TGCCACTGCTTTGATGACCC, Fw2.2-R:

TATAACGCACCTCTACCCGC. Thermocycling conditions were $95^{\circ} \mathrm{C}$ for $1 \mathrm{~min}$, followed by 40 cycles of $95^{\circ} \mathrm{C}$ for $10 \mathrm{~s}, 55^{\circ} \mathrm{C}$ for $5 \mathrm{~s}$, and $72^{\circ} \mathrm{C}$ for $15 \mathrm{~s}$. Relative gene expression data were analyzed using the $2^{-}$ $\triangle \Delta \mathrm{CT}[32]$.

\section{Results}

Analysis of fw2.2 gene structure and phylogenetic analysis of Lycium ruthenicum 
The full length of the DNA of Lycium ruthenicum fw2.2 is $2323 \mathrm{bp}$, it is divided into three exon regions by introns, the lengths of which are exon $1: 259 \mathrm{bp}$, exon 2:210bp, exon $3: 80 \mathrm{bp}$. The cDNA sequence is $549 \mathrm{bp}$ in length, with a total coding of 182 amino acids. The fw2.2 gene of Lycium ruthenicum has a PLAC8 (Placenta-specific 8) conserved domain ( Fig. 1a), and belongs to the same family as fw2.2 of other plants. After BLASTp alignment on $\mathrm{NCBI}$, some homologous protein sequences are obtained. Most of them were FWL/CNR family proteins, and few of them are PCR (Plant cadmium resistance) family proteins ( Fig. 1b). The two family proteins have the same conserved domain PLAC8 and similar structures. The selected Solanaceae fw2.2 homologous protein including Lycium ruthenicum fw2.2, Lycopersicon fw2.2, Physalis POS2, and Capsicum CNR1 are clustered in the same branch, and the amino acid sequence similarity is more than $85 \%$ ( Fig. 1C), indicating that fw 2.2 protein is relatively conserved in Solanaceae. FW2.2-like genes have been renamed as the Cell Number Regulator (CNR) family, and it has a negative regulatory effect on cell number $[17,18]$. Studies have shown that $f w 2.2$ can explain $30 \%$ and $47 \%$ of the fruit size phenotypic variation in Lycopersicon pimpinellifolium and Lycopersicon pennellii, respectively[19]. The POS2 (physalis organ size 2) in Physalis floridana encodes a putative ortholog of $f w 2.2$, which can regulate the cell cycle and has a negative effect on fruit size[20]. The function of CNR1 in pepper is unknown. Tomato fw2.2 and Physalis POS2 both have the functions of regulating cell division and affecting fruit size. It is speculated that Lycium ruthenicum fw2.2 which has a close phylogenetic relationship with them may also have similar functions.

\section{Establishment of Lycium ruthenicum regeneration system}

The seeds of Lycium ruthenicum are disinfected and inoculated on 1/2 MS medium, we have established a suitable regeneration system of Lycium ruthenicum after the induction and differentiation of callus, and the rooting of regenerated seedlings (Additional file 1 Table S1). Although the callus induction rate in each medium is $100 \%$ after 15 days of callus induction, the callus growth status is different. Among them, callus grows best on A3 medium (MS $+0.5 \mathrm{mg} / \mathrm{L} 6-\mathrm{BA}+0.5 \mathrm{mg} / \mathrm{L} \mathrm{NAA}$ ) with green appearance, loose structure, no browning, and low vitrification, which is the most suitable medium (Additional file 1 Table S1). The callus was subsequently transferred to the medium B1-B10 to induce differentiation, and the differentiation rate and multiplication coefficient were counted after 30 days. We found that the callus did not differentiate or the differentiation rate was low when it was cultured on the medium without 6-BA (B5) or the concentrations of 6-BA were higher (B1-B4 ). Both the differentiation rate and the multiplication coefficient were significantly increased on the medium supplemented with low concentration of 6-BA (less than $0.5 \mathrm{mg} / \mathrm{L}$ ). When the callus was cultured on $\mathrm{B} 7$ ( $\mathrm{MS}+0.2 \mathrm{mg} / \mathrm{L} \mathrm{6-BA}+$ $0.05 \mathrm{mg} / \mathrm{L} \mathrm{NAA}$ ) medium for cluster shoot induction, the differentiation rate and multiplication coefficient were the highest, which were $(95.83 \pm 2.23) \%$ and $(7.06 \pm 0.22) \%$ respectively. The callus did not brown and the degree of vitrification was relatively low (Additional file 1 Table S1). The differentiated shoots were then inoculated on 1/2 MS medium without hormones, and rooting can be induced after 15 days.

\section{Target site selection and sgRNA Design}


The CRISPR online design tool (http://crispr.dbcls.jp/ ) was used to determine the location of the target, the genome of tomato ( Solanum lycopersicum genome, SL2.40), a species with high homology with Lycium ruthenicum, was used as a reference to improve target specificity. Based on the selection criteria that the PAM site sequence is NGG, the GC content is $40 \%-70 \%$, the target sequence avoids spanning intron regions and the occurrence of more than 4 consecutive $T$ bases, the top sgRNA were selected for the knockout experiments. The sgRNA used in this study are shown in Table S2. The position of fw2.2-1 and fw2.2-2 is 1857-1879 and 171-193 respectively, spanning 1664 bases.

\section{Establishment of Lycium ruthenicum genetic transformation system and efficiency evaluation of CRISPR/Cas}

Two single sgRNAs and a dual sgRNAs (sgRNA1 and sgRNA2) of fw2.2 were designed that target different sites after the whole sequence being cloned and phylogenetic analyzed. The target site of fw2.2sgRNA1 and fw2.2-sgRNA2 is located in exon 2 and exon 1 respectively (Fig. 2a). The high efficient Agrobacterium tumefaciens-mediated transformation of black wolfberry was performed here using the leaves under the condition of 0.2 Agrobacterium concentration (OD600), 10 min infection time, 200 $\mu \mathrm{mol} / \mathrm{L}$ acetosyringone concentration and $2 \mathrm{~d}$ co-culture time (Additional file $1 \mathrm{Table} \mathrm{S3).} \mathrm{The} 40 \mathrm{mg} / \mathrm{L}$ hygromycin selection marker plus $200 \mathrm{mg} / \mathrm{L}$ carbenicillin were used to inhibit Agrobacterium growth. The entire experimental cycle took approximately 2 months from incubation to mutant identification with $2 \mathrm{~d}$ co-cultivation, $15 \mathrm{~d}$ calli production, $30 \mathrm{~d}$ differentiation and sub-culture (Fig. $2 \mathrm{~b}$ ). The details of the transformation media can be found in Fig. 2c. The mean transformation efficiencies of these three lines were $2.66 \%, 1.18 \%$ and $5.33 \%$, respectively.

In this study, a lot of resistant seedlings were produced. Twenty-one out of twenty-two, six out of eleven and fifteen out of sixteen bigger plants were detected with novel mutations in the sgRNA1, sgRNA2 and sgRNA1/sgRNA2 regions. As shown in Fig. 2d, the gene editing efficiency of fw2.2-1 target is high (95.45\%), while that of fw2.2-2 target is low (54.55\%). However, the editing efficiency of homozygous mutants (18.18\%) and biallelic mutants (9.09\%) is higher than those of fw2.2-1 (4.55\%).

The dual-sgRNA CRISPR/Cas9 system was highly reproducible and highly efficient since it could result in more reliable loss-of-function alleles that lack a large essential part of the gene[21]. Here we found the editing efficiency of $f w 2.2$ in the homozygote/biallelic mutations altogether $(56 \%)$ by the dual-sgRNA CRISPR/Cas9 system is more than twice (27\%) of that by the sgRNA CRISPR/Cas9 system though the editing efficiency of the dual-sgRNA system is only $93.75 \%$,which is a little less than that of $(95.45 \%)$ the sgRNA1 system (Fig. 2e\&f). It was also found that there was a $1281 \mathrm{bp}$ segment deleted and 29bp insertion in the fw2.2 of a T0 plant (Fig. 2g), which is similar to the result such as 934-bp deletion mutation at the AtMIR169a locus of Arabidopsis[21] .

\section{Expression analysis of fw2.2 in geneedited seedlings by quantitative real-time PCR}

We found that the expression of the fw2.2 gene changed significantly (Fig. 3). Among the selected geneedited seedlings, the expression of fw2.2 gene decreased most significantly in X21, which had a large 
deletion of $1281 \mathrm{bp}$, and its expression was only 0.01 . X9 is a homozygous mutant, the expression of fw2.2 gene in which was only 0.03 , and followed by $\mathrm{X} 17$ and $\mathrm{X} 20$, which were 0.06 and 0.15 respectively. When a gene expression cassette is introduced into a genome by CRISPR/Cas9, the sgRNA target gene becomes inactive because of disruption of the gene, which probably influences the gene expression. These data suggest that CRISPR/Cas 9 applied to $f w 2.2$ had a significant effect on reducing gene expression, which probably influences the developmental characteristics of the modified strain if the inactive gene is generally involved in metabolism.

\section{Discussion}

Until now, successful genome editing mediated by CRISPR/Cas9 was demonstrated in Solanaceae, including tomato[22], potato[23], tobacco[24], Petunia[25], Physalis[14] and eggplant[26], fully demonstrating the broad prospects of molecular genetic technology for crop improvement. However, due to the complex genomes, slow growth cycles, difficulties of transformation and a lack of genomic information, it remains a long and arduous task to apply CRISPR/Cas9 to Lycium ruthenicum. In this study, we established a suitable regeneration and genetic transformation system for Lycium ruthenicum, which laid the foundation for the subsequent successful transformation of CRISPR/Cas 9 plasmids. Studies have shown that simultaneous targeting of multiple regions of the same gene or functionallyredundant genes can improve gene editing efficiency[27]. We constructed double target editing vector pCAMBIA1300-fw2.2-1/2 except for the single-target editing vectors pCAMBIA1300-fw2.2-1 and pCAMBIA1300-fw2.2-2. And both target sites were selected by the CRISPR online design tool using the tomato genome as a reference to improve specificity. Different mutation including homozygous, biallelic and heterozygous were obtained, and it was found that the mutation type with the highest proportion was 3 base deletion occurring at 3-5bp upstream of PAM site (Fig. 2h). The deletion of the $1281 \mathrm{bp}$ large fragment and 29bp insertion in the fw2.2 (Fig. 2g) were also found in the T0 plant. All these results suggest that the CRISPR/Cas9 system applied to Lycium ruthenicum is effective.

A recent report has demonstrated $f w 2.2$ which encodes a negative cell number regulator increases fruit weight by approximately $30 \%$ between the domesticated tomato and its wild relatives in the genus[5]. The overexpression of ZmCNR1 and ZmCNR2 (members of the fw2.2 gene family in corn ) resulted in the decrease in plant and organ size[28]. The developmental expression profile of Pafw2.2-like, a fw2.2/CNR family member in avocado revealed that the transcript levels of Pafw2.2-like were markedly higher in small fruit tissues than in normal fruit tissues[12]. Scorza et al.[8] found that the large-fruited peach exhibited a higher number of cells at all developmental stages comparing with small-fruited peach. Olmstead et al. [9]reported that the increases in cell number rather than cell size resulted in the difference of cherry fruits in the process of domestication and modern breeding. Franceschi et al.[13] identified the homologous genes PpCNR12 and PpCNR20 of fw2.2 in cherry and speculated that they were involved in fruit size regulation. More and more studies have supported that fw2.2 plays an important role in fruit size. In our study, the fw2.2 gene of Lycium ruthenicum that we identified has high homology with other species, studies have shown that specific $f w 2$.2-like proteins might share a similar biological function with the tomato $f w 2.2[28,29]$, which suggested that $f w 2.2$ in Lycium ruthenicum might be associated to 
regulation and/or repression of fruit cell division. Whether we could produce the bigger fruit phenotypes of black wolfberry or not needs to wait until next summer.

\section{Conclusion}

This study is the first to use CRISPR/Cas9 technology for targeted genome editing in Lycium ruthenicum, and demonstrates that the system has high editing efficiency. The results obtained in this study can help to understand the mechanism of fruit development. Meanwhile, the regeneration and genetic transformation system established in this study can provide theoretical guidance for transgenic research of Lycium ruthenicum. The targeted knockout of desired genes using CRISPR/Cas9 is also of great significance for developing new traits of Lycium ruthenicum. The effect of successful editing on fruit development needs further morphological analysis and transcriptome sequencing.

\section{Declarations}

\section{Acknowledgements}

The authors thank The World-Class Discipline Construction and Characteristic Development Guidance Funds for Beijing Forestry University (2019XKJS0323) for providing funding for this research. We thank Associate Prof. Yuming Lu in Shanghai Center for Plant Stress Biology for providing pCAMBIA1300-Cas 9 binary vector in establishing wolfberry CRISPR/Cas9 system. The authors thank Prof. Chaoying He in Institute of Botany for helpful discussions on fw2.2.

\section{Authors' contribution}

H. Z. designed the experiments which were conducted by J. L., H. W., W.W. and T. L.. J.L performed the editing experiments. H. W. helped with designing the sgRNAs, constructing the vectors and making the photography. W.W. helped with analysis of the results and T.L. did the regeneration at first. All authors read and approved the submission of this manuscript.

\section{Funding}

This project was financially supported by The World-Class Discipline Construction and Characteristic Development Guidance Funds for Beijing Forestry University (2019XKJS0323).

\section{Availability of data and materials}

We provide supporting and necessary data for publication of the article. All supporting data is present in the article and Additional files. The strains and plasmids associated with this work will be made physically available by the authors upon reasonable request.

\section{Ethics approval and consent to participate}


Not applicable.

Consent for publication

Not applicable.

Conflict of interest

The authors declare no conflict of interest.

\section{References}

1. Zheng J, Ding C, Wang L, Li G, Shi J, Li H, Wang H, Suo Y. Anthocyanins composition and antioxidant activity of wild Lycium ruthenicum Murr. from Qinghai-Tibet Plateau. Food Chemistry. 2011; 126:859865.

2. Zeng S, Wu M, Zou C, Liu X, Shen X, Hayward A, Liu C, Wang Y. Comparative analysis of anthocyanin biosynthesis during fruit development in two Lycium species. Physiologia Plantarum. 2014; 150:505516.

3. Grandillo S, Ku HM, Tanksley SD. Identifying the loci responsible for natural variation in fruit size and shape in tomato. Theoretical and Applied Genetics. 1999; 99:978-987.

4. Paran I, van der Knaap E. Genetic and molecular regulation of fruit and plant domestication traits in tomato and pepper. Journal of Experimental Botany. 2007; 58:3841-3852.

5. Frary A, Nesbitt TC, Grandillo S, Knaap E, Cong B, Liu J, Meller J, Elber R, Alpert KB, Tanksley SD. fw2.2: a quantitative trait locus key to the evolution of tomato fruit size. Science (New York, NY). 2000; 289:85-88.

6. Tanksley SD. The Genetic, Developmental, and Molecular Bases of Fruit Size and Shape Variation in Tomato. The Plant Cell. 2004; 16:S181.

7. Cong B, Liu J, Tanksley SD. Natural alleles at a tomato fruit size quantitative trait locus differ by heterochronic regulatory mutations. Proceedings of the National Academy of Sciences. 2002; 99:13606.

8. Scorzal R, May LG, Purnell B, Upchurch B. Differences in Number and Area of Mesocarp Cells between Small- and Large-fruited Peach Cultivars. Am Soc Hortic Sci. 1991; 116:861-864.

9. Olmstead JW, lezzoni AF, Whiting MD. Genotypic Differences in Sweet Cherry Fruit Size are Primarily a Function of Cell Number. 2007; v. 132.

10. Rapoport HF, Manrique T, Gucci R. Cell division and expansion in the olive fruit. 2004.

11. Harada T, Kurahashi W, Yanai M, Wakasa Y, Satoh T. Involvement of cell proliferation and cell enlargement in increasing the fruit size of Malus species. Scientia Horticulturae. 2005; 105:447-456.

12. Dahan Y, Rosenfeld R, Zadiranov V, Irihimovitch V. A proposed conserved role for an avocado fw2.2 like gene as a negative regulator of fruit cell division. Planta. 2010; 232:663-676. 
13. De Franceschi P, Stegmeir T, Cabrera A, van der Knaap E, Rosyara UR, Sebolt AM, Dondini L, Dirlewanger E, Quero-Garcia J, Campoy JA et al. Cell number regulator genes in Prunus provide candidate genes for the control of fruit size in sweet and sour cherry. Molecular Breeding. 2013; 32:311-326.

14. Lemmon ZH, Reem NT, Dalrymple J, Soyk S, Swartwood KE, Rodriguez-Leal D, Van Eck J, Lippman ZB. Rapid improvement of domestication traits in an orphan crop by genome editing. Nature Plants. 2018; 4:766-770.

15. Xu J, Hua K, Lang Z. Genome editing for horticultural crop improvement. Horticulture Research. 2019; 6:113.

16. Gianoglio S, Comino C, Moglia A, Lanteri S, Jaime P, Diego O. CRISPR/Cas9-mediated Knock-out of Polyphenol Oxidase Genes in Eggplant (Solanum melongena L.). UniversitatPolitècnica de València. 2017; 159.

17. Xu J, Xiong W, Cao B, Yan T, Luo T, Fan T, Luo M. Molecular characterization and functional analysis of "fruit-weight2.2-like" gene family in rice. Planta. 2013; 238:643-655.

18. Monforte AJ, Diaz A, Caño-Delgado A, van der Knaap E. The genetic basis of fruit morphology in horticultural crops: lessons from tomato and melon. Journal of Experimental Botany. 2014; 65:46254637.

19. Alpert KB, Grandillo S, Tanksley SD. fw2.2: a major QTL controlling fruit weight is common to both red- and green-fruited tomato species. Theoretical \& Applied Genetics. 1995; 91:994-1000.

20. Li Z, He C. Physalis floridana Cell Number Regulator1 encodes a cell membrane-anchored modulator of cell cycle and negatively controls fruit size. Journal of Experimental Botany. 2015; 66:257-270.

21. Zhao Y, Zhang C, Liu W, Gao W, Liu C, Song G, Li W-X, Mao L, Chen B, Xu Y et al. An alternative strategy for targeted gene replacement in plants using a dual-sgRNA/Cas9 design. Scientific Reports. 2016; 6:23890.

22. Brooks C, Nekrasov V, Lippman ZB, Van Eck J. Efficient Gene Editing in Tomato in the First Generation Using the Clustered Regularly Interspaced Short Palindromic Repeats/CRISPRAssociated9 System. Plant Physiology. 2014; 166:1292.

23. Wang S, Zhang S, Wang W, Xiong X, Meng F, Cui X. Efficient targeted mutagenesis in potato by the CRISPR/Cas9 system. Plant Cell Reports. 2015; 34:1473-1476.

24. Nekrasov V, Staskawicz B, Weigel D, Jones JDG, Kamoun S. Targeted mutagenesis in the model plant Nicotiana benthamiana using Cas9 RNA-guided endonuclease. Nature Biotechnology. 2013; 31:691693.

25. Zhang B, Yang X, Yang C, Li M, Guo Y. Exploiting the CRISPR/Cas9 System for Targeted Genome Mutagenesis in Petunia. Scientific Reports. 2016; 6:20315.

26. Maioli A, Gianoglio S, Moglia A, Acquadro A, Valentino D, Milani AM, Prohens J, Orzaez D, Granell A, Lanteri S et al. Simultaneous CRISPR/Cas9 Editing of Three PPO Genes Reduces Fruit Flesh Browning in Solanum melongena L. Frontiers in plant science. 2020; 11:607161. 
27. Xie K, Minkenberg B, Yang Y. Boosting CRISPR/Cas9 multiplex editing capability with the endogenous tRNA-processing system. Proceedings of the National Academy of Sciences. 2015; 112:3570.

28. Guo M, Rupe MA, Dieter JA, Zou J, Spielbauer D, Duncan KE, Howard RJ, Hou Z, Simmons CR. \&lt;em\&gt;Cell Number Regulator1\&lt;/em\&gt; Affects Plant and Organ Size in Maize: Implications for Crop Yield Enhancement and Heterosis. The Plant Cell. 2010; 22:1057.

29. Libault M, Zhang X-C, Govindarajulu M, Qiu J, Ong YT, Brechenmacher L, Berg RH, Hurley-Sommer A, Taylor CG, Stacey G. A member of the highly conserved FWL (tomato FW2.2-like) gene family is essential for soybean nodule organogenesis. The Plant Journal. 2010; 62:852-864.

30. Bateman A, Coin L, Durbin R, Finn RD, Hollich V, Griffiths-Jones S, Khanna A, Marshall M, Moxon S, Sonnhammer ELL et al. The Pfam protein families database. Nucleic Acids Research. 2004; 32:D138D141.

31. Marchler-Bauer A, Lu S, Anderson JB, Chitsaz F, Derbyshire MK, DeWeese-Scott C, Fong JH, Geer LY, Geer RC, Gonzales NR et al. CDD: a Conserved Domain Database for the functional annotation of proteins. Nucleic Acids Research. 2011; 39:D225-D229.

32. Livak KJ, Schmittgen TD. Analysis of Relative Gene Expression Data Using Real-Time Quantitative PCR and the 2- $\Delta \Delta C T$ Method. Methods. 2001; 25:402-408.

\section{Figures}


a
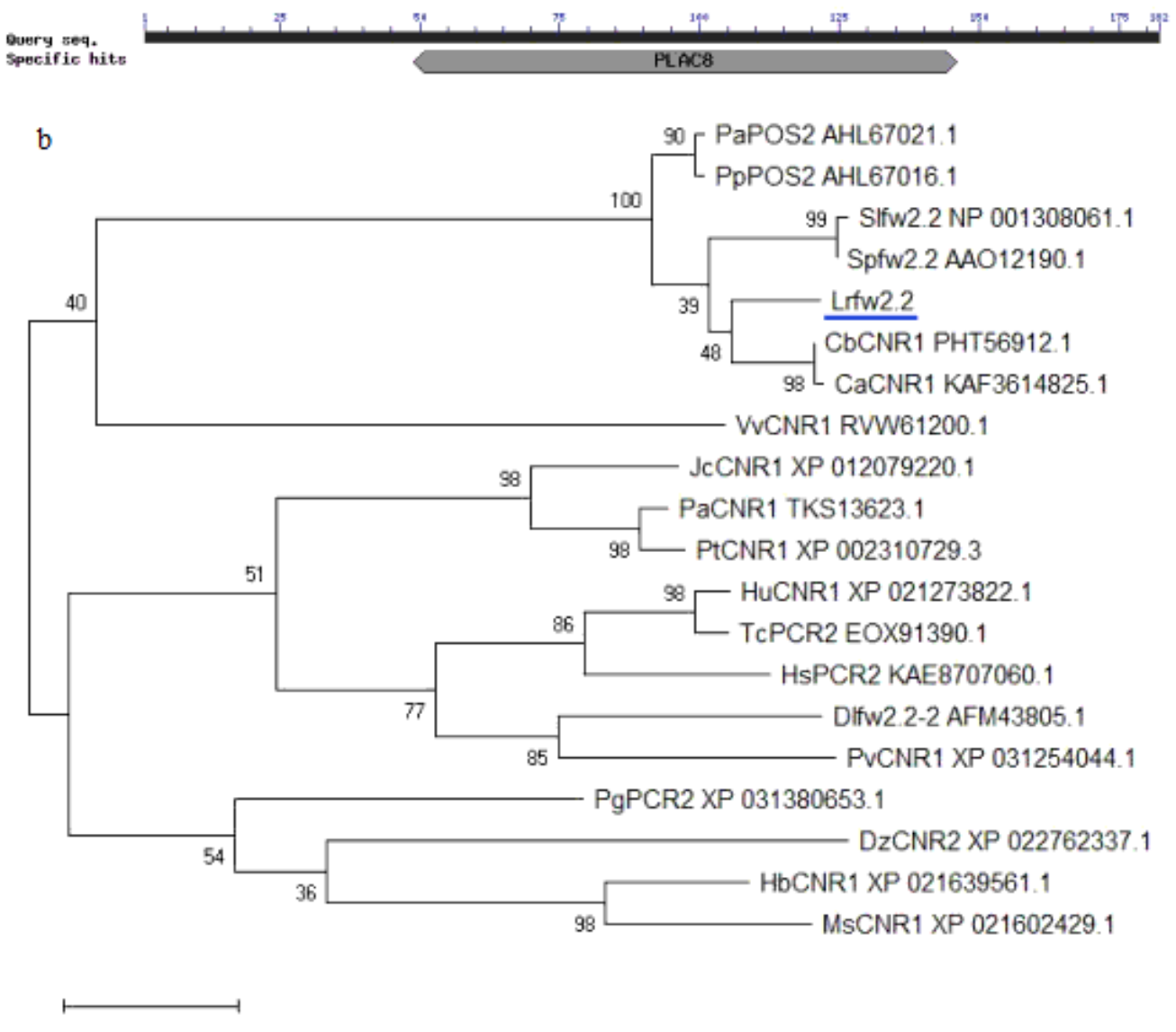

010

C

[Lycium-ruthenicum] [Solanum-1yeopersieum]

[Solanum-penne1111]

[Capsieum-annuum]

[Physa11s-pruinosa]

[Lycium-ruchenicum]

[Solanum-1ycopersacum]

[Solanum-penne1111]

[Capsicum-anntum]

[Physal1s-pruinosa]

[Iyeium-ruthenieum]

[Solanum-1ycopersicur]

[Solanum-penne11i1]

[Capsi cum-annulam]

[Physalis-pruinosa]

[Iycium-ruthenicum]

[Solanum-1yoopersicum]

[Solanum-pennel1ii]

[Capsicum-annuum]

[Physalis-pruinosa]

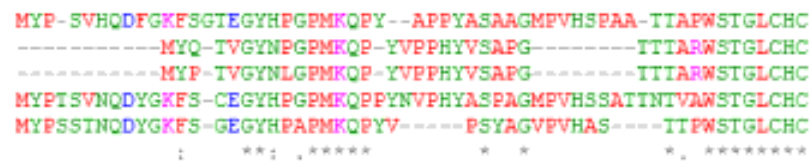

FDDPANCLVICVCPCITFGQISEILNRGTTSCAGRGNYYLLIGLTGEPSLYSCFYRSKMR FDDPAWCLVT SVCPCITFGOTSEILNKGTTSCGSRGALYCLLSLTGLPSLYSCFYRSKMA FDDPANCLVISVCPCITFGQISEILNKGI ISCGSRGALYCLIGLTGLPSLYSCFYRSKME FDDPAWCLVTCVCPCITFGQISEILNKGITSCASRGALYGLLGVTGLPSLYSCFYRSKME FDDPANCLVTCVCPCIIFGQTSEILNRGITSCASRGALYGLLALTGLPSLYSCFYRSKYM

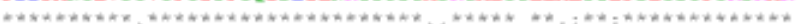

GQYDLEERPCVDCLVHVFCEPCALCQEYRELRIRGFDMGIGWQAMIERQSRGGAVTMPEY GOYDLEEAPCVDCLVHVFCEPCALCOEYRELRORGGDMGIGWOANMDAOSRG--VTMPEY

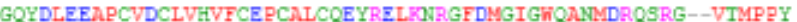
GOYDLEEAPCVDCLVHVFCEPCALCOEYRELKOTRGFDMGIGWOANMEROSRG--VTMPPY GQYDLEEAPCVDCLVHVFCEPCNLCQEYRELRONRGFDMGIGWOANIERQSRG--VTMPPC

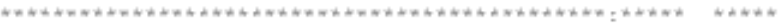

HASTTR $\quad 182$

HAGITR 163

HAGIMTR 163

NAGTR 193

HAGITR $\quad 174$

\section{Figure 1}

Conserved domain and phylogenetic analysis of Lycium ruthenicum fw2.2. a. Conserved domain analysis of fw2.2. b. Phylogenetic tree of the predicted CNR/FWL proteins, the plants corresponding to the CNR/FWL proteins are: PpPOS2, Physalis pruinosa; PaPOS2, Physalis aequata; Slfw2.2, Solanum lycopersicum; Spfw2.2, Solanum pimpinellifolium; CaCNR1, Capsicum annuum; CbCNR1, Capsicum Baccatum; VvCNR1, Vitis vinifera; JcCNR1, Jatropha curcas; PaCNR1, Populus Alba; PtCNR1, Populus 
trichocarpa; HuCNR1, Herrania umbratica; TcPCR2, Theobroma cacao; HsPCR2, Hibiscus syriacus; DIfw2.2-2, Dimocarpus longan; PvCNR1, Pistacia vera; PgPCR2, Punica granatum; DzCNR2, Durio zibethinus; HbCNR1, Hevea brasiliensis; MsCNR1, Manihot esculenta. c. Multiple sequence alignment of fw2.2 gene of Lycium ruthenicum and other species in Solanaceae, the gene accession number are: Solanum lycopersicum: NP_001308061.1; Solanum pennellii: AA012196.1; Capsicum annuum: PHT91598.1; Physalis pruinosa: AHL67016.1.
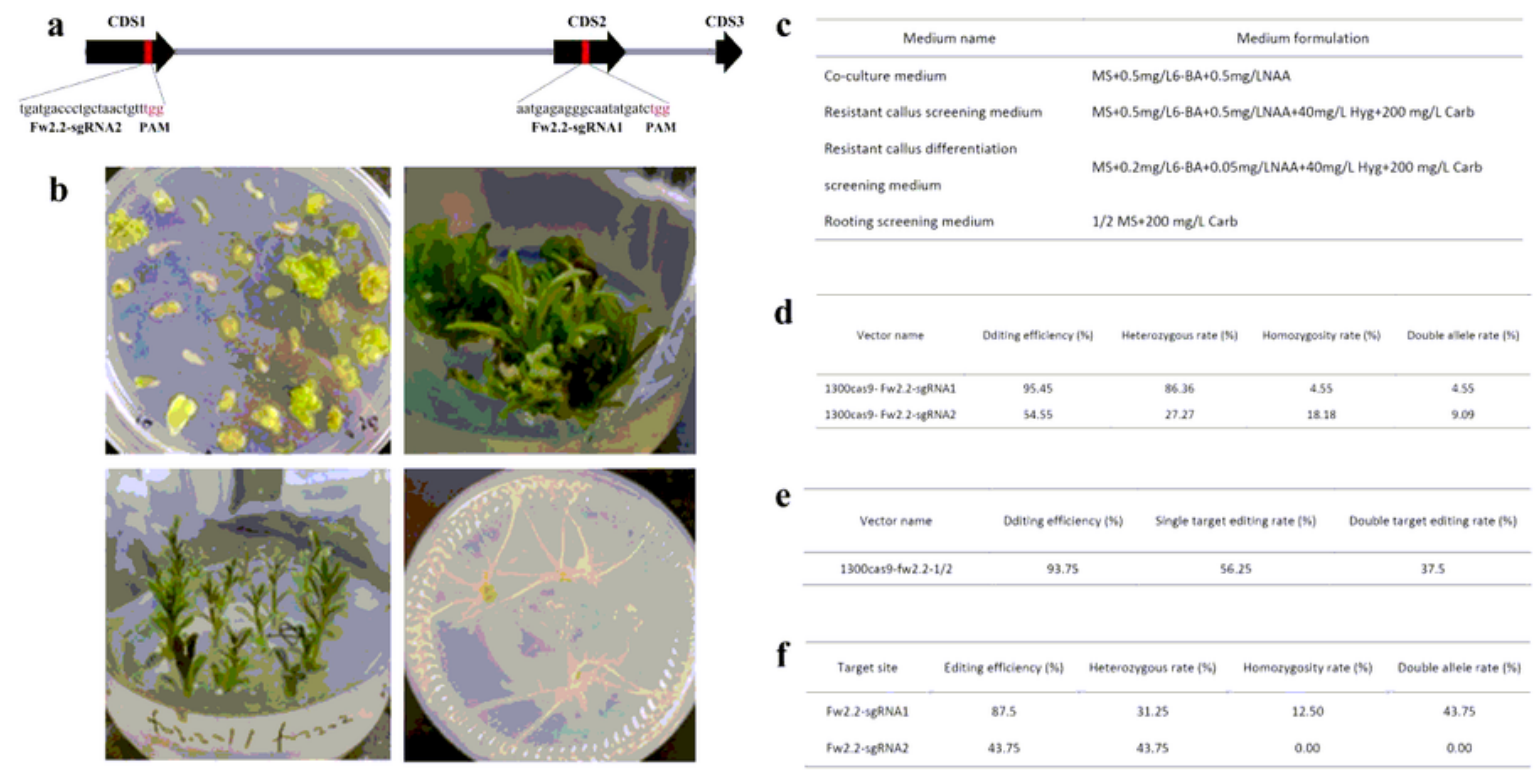

d
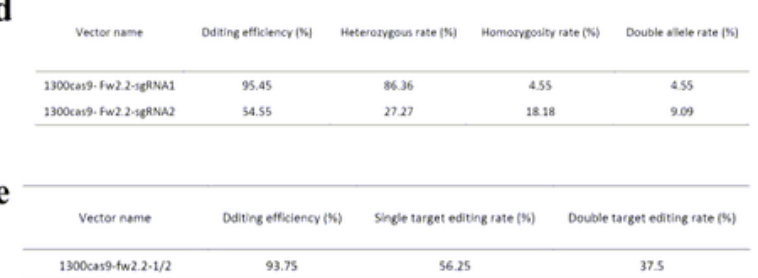

\begin{tabular}{|c|c|c|c|c|}
\hline Target site & Editing efficiency (s) & Heteroarygous rate (S) & Homorygosity rate (s) & Double allele rate $(\mathrm{S})$ \\
\hline FW22:-39 & 87.5 & 31.25 & 12.50 & 43.75 \\
\hline FW2.2-SERNA2 & 43.75 & 43.75 & 0.00 & 0.00 \\
\hline
\end{tabular}

Fw2.2-sgRNA2

atgtatccatcggttcat pagacttcggtaaattctctggtactgaagggtatcatccaggccctatgaaacaaccttatgctcetcectatgcatctgccgccggtatgecagtgcactctcaggcagccaccacggcgecgtggtcaaccggtctt

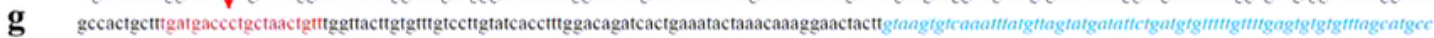

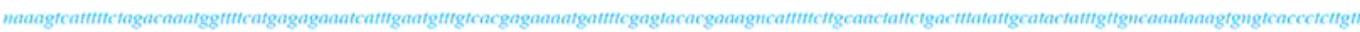

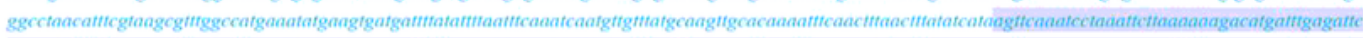

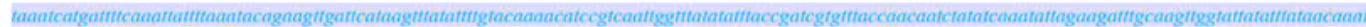

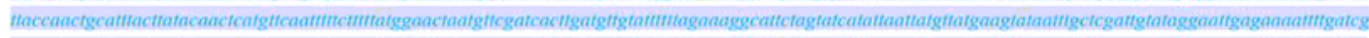

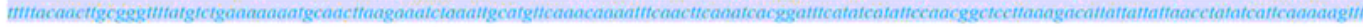

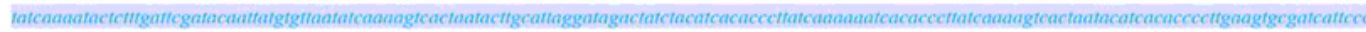

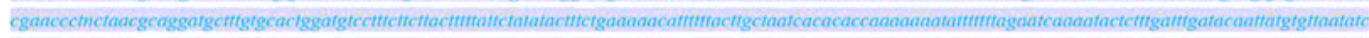

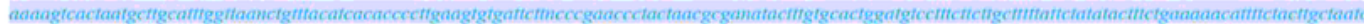

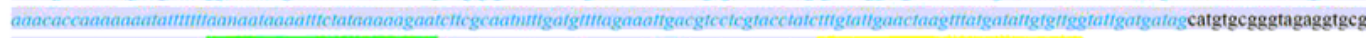

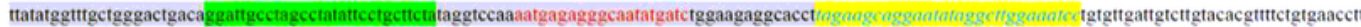

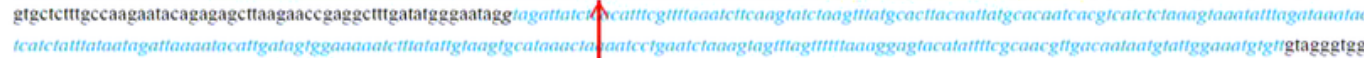
caagctaatatggaaagacaaagcaggggaggggcagttacgatgcccccttatcatgcaggcat foccaggtgaaa Fw2.2-sgRNA1

$$
\text { fw2.2-1 }
$$

h AAAATGAGAGgGCAATATGATCTGGAaGAGgCACCTTGTGTTGATT WT AAAATGAGAGGGCAATATGAATCTGGAAGAGGCACCTTGTGTTGATT $+1(\times 6)$ AAAATGAGAGGGCAATATGTATCTGGAAGAGGCACCTTGTGTTGATT $+1(\times 1)$ AAAATGAGAGGGCAATATGAGTCTGGAAGAGGCACCTTGTGTTGATT $+1(\times 1)$ AAAATGAGAGGGCAATAT...ATCTGGAAGAGGCACCTTGTGTTGATT $-1(\times 1)$ AAAATGAGAGGGCAATAT-CTGGAAGAGGCACCTTGTGTTGATT $-3(\times 13)$ TCTGGT $-4(\times 1)$ ( AAAATGAGAGGG GGCACCTTGTGTTGATT $-9(\times 1)$

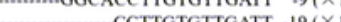
(1)

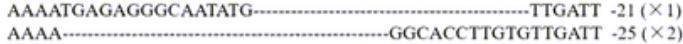
AAAA-

fw $2.2-2$

TTTGATGACCCTGCTAACTGTTTGGTTACTTGTGTTTGTCCTT WT TTTGATGACCCTGCTAACTCTGTTTGGTTACTTGTGTTTGTCCTT $+2(\times 1)$ TTTGATGACCCTGCTAACTTGTTTGGTTACTTGTGTTTGTCCTT $+1(\times 4)$ TTTGATGACCCTGCTAACCTGTTTGGTTACTTGTGTTTGTCCTT $+1(\times 1)$ TTTGATGACCCTGCTAACTCGTTTGGTTACTTGTGTTTGTCCTT $+1(\times 1)$ TTTGATGACCCTGCTA.......-GTTTGGTTACTTGTGTTTGTCCTT $-3(\times 1)$ TTTGATGACCCTGCTAACT-....................TGTGTTGTCCTT $-11(\times 2)$

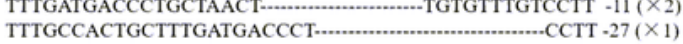

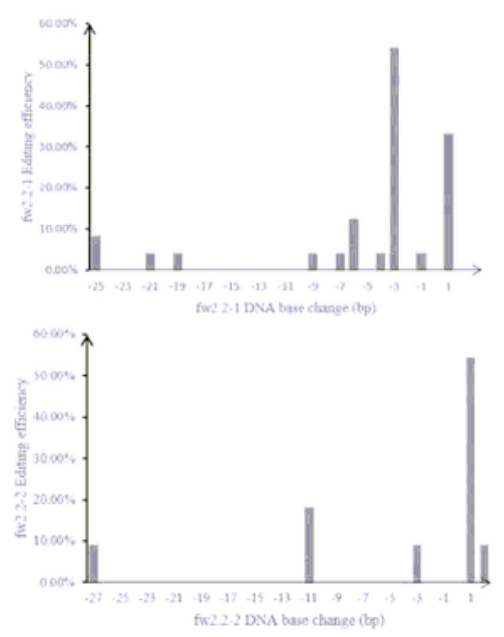


Genome editing in Lycium ruthenicum using CRISPR/Cas9 technology. a. fw2.2 gene structure and sequences of the target sites. Black boxes: exons; grey lines: introns; sgRNA target sites and the PAM regions (Red). b. The process of transformation (Two-week-old callus; differential shoots after four-week of subculture; elongated shoots after six-week of subculture; eight-week-old rooted transformant). c. The media information used in the transformation. $d$. The gene editing rate of different fw2.2 target sites in Lycium ruthenicum. e. The editing efficiency of the dual-sgRNA system. $f$. Comparison of editing rate between constructs with different number of sgRNA cassettes. g. The combination of large fragment deletions with insertions in a T0 plant. The $1515 \mathrm{bp}$ in italic is the intron. $1281 \mathrm{bp}$ deletions are labeled in gray. $29 \mathrm{bp}$ insertions shown in yellow are almost the inversion of the fragment nearby labeling with green. h. Editing type and preference of different target sites.

1.20

1.00

0.80

0.60

0.40

0.20

0.00
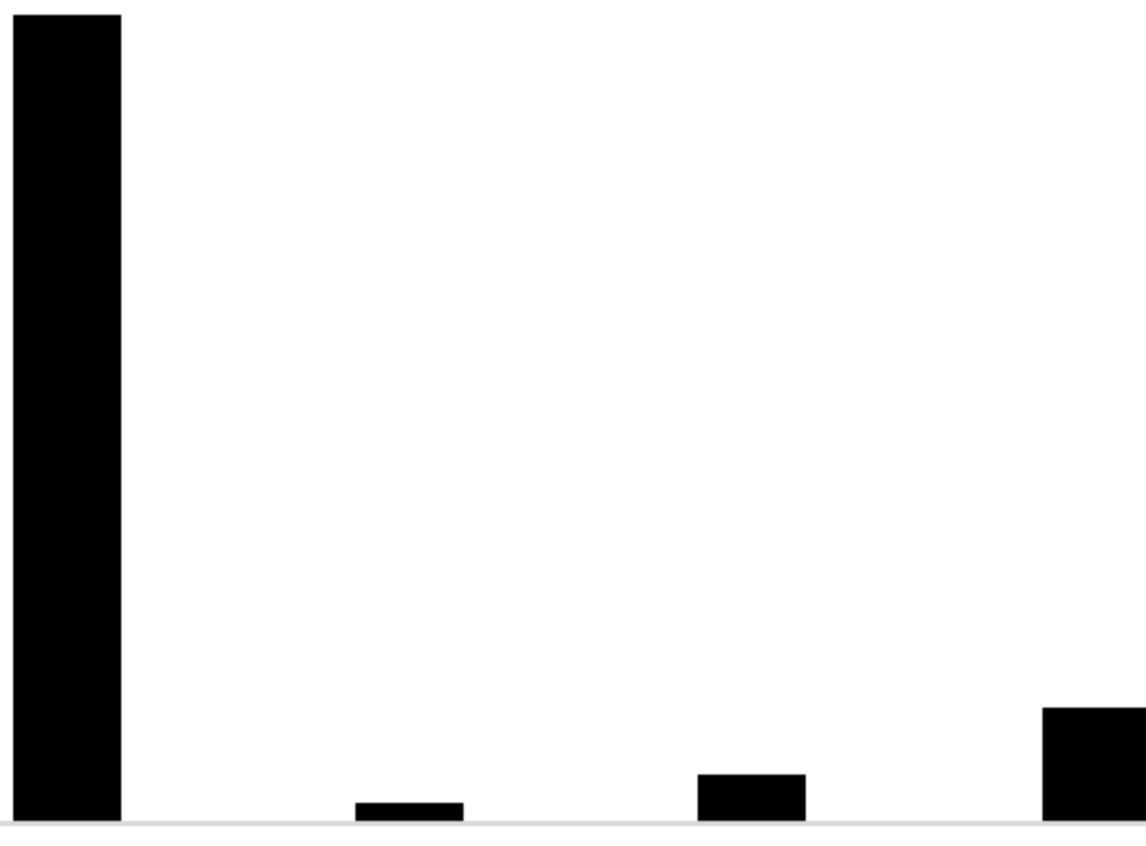

CK

$\mathrm{X} 9$

$\mathrm{X} 17$

$\mathrm{X} 20$

$\mathrm{X} 21$

Figure 3

Verification of the relative expression of fw2.2 in gene-edited seedlings 


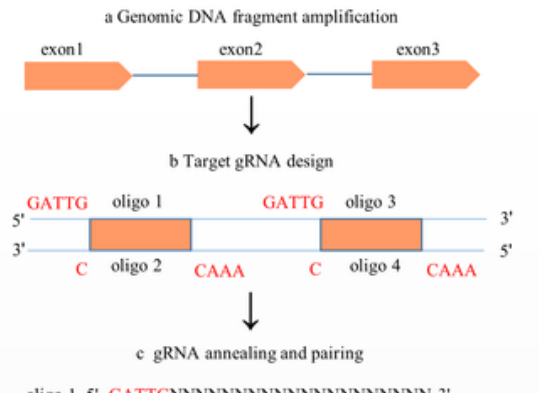

oligo $15^{\circ}$-GATTGNNNNNNNNNNNNNNNNNNNN-3'

oligo $23^{\prime} \cdot \quad \|_{\text {CNNNNNNNNNNNNNNNNNNNCAAA-5' }}$
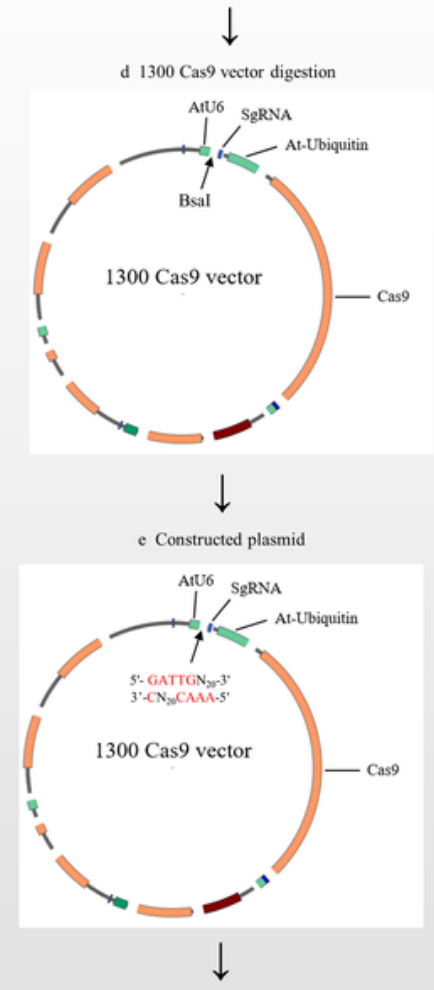

f Agrobacterium transformation into Lycium ruthenicum leaves

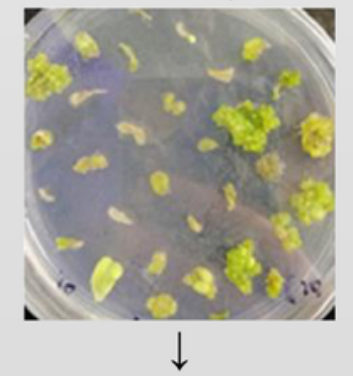

$\mathrm{g}$ Callus selection, bud selection, rooting selection $\downarrow$

h Target site mutation verification

\section{Figure 4}

Single target vector construction 


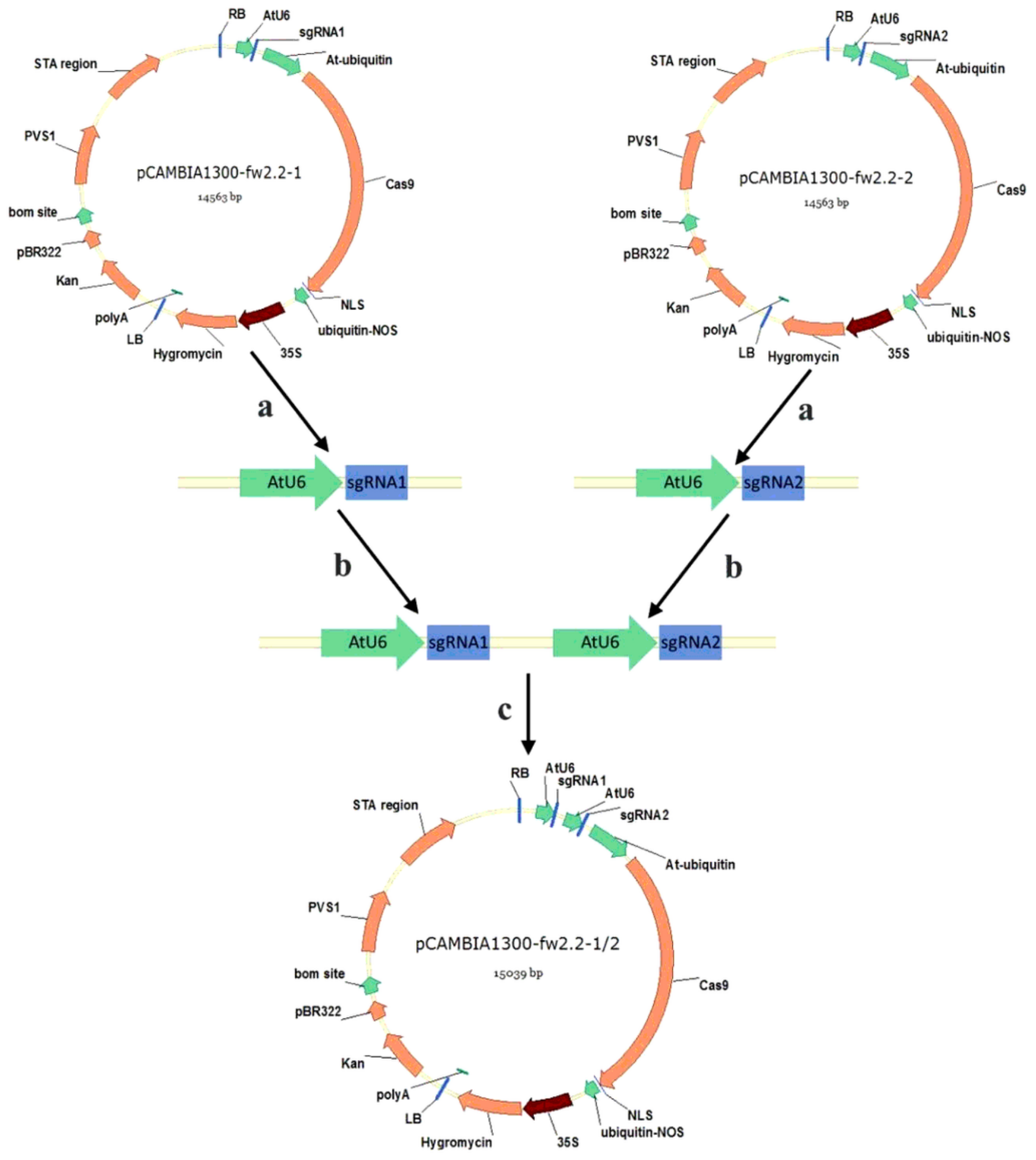

\section{Figure 5}

Dual target vector construction 
原序列 TTTGATGACCCTGCTAACT--GTTIGGTTACTTGTGTTT 染色体1 TTTGATGACCCTGCTAACT--GTTTGGTTACTTGTGTTT 染色体2 TTTGATGACCCTGCTAACTTGTTTGGTTACTTGTGTTT +

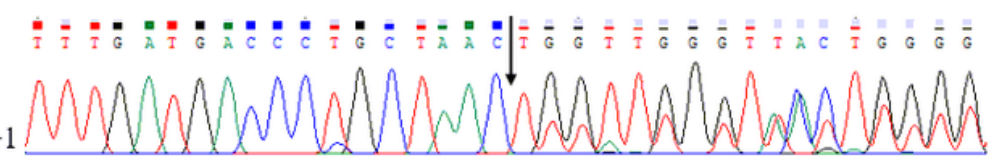

靶点Fw2.2-2

原序列 TTTGATGACCCTGCTAACTGTTTGGTTACTTGTGTTT 染色体1 TTTGATGACCCTGCTAACT 染色体2 TTTGATGACCCTGCTAACT-TGTGTTT -11

靶点FW2.2-2

原序列 AAGGACAAACACAAGTAACCAAAC--AGTTAGCAGG 染色体1 AAGGACAAACACAAGTAACCAAACGAGTTAGCAGG + 1 染色体2 AAGGA-
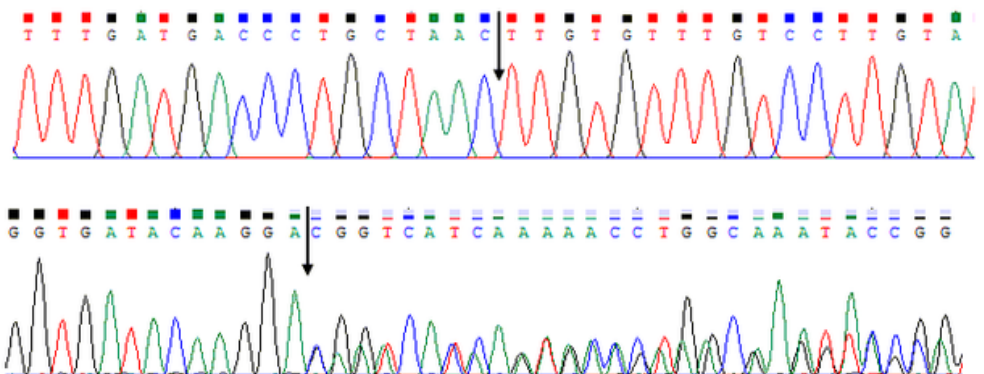

\section{Figure 6}

Mutation types and sequencing chromatograms of gene-edited seedlings.

\section{Supplementary Files}

This is a list of supplementary files associated with this preprint. Click to download.

- Additionalfile1.docx

- Additionalfile2.docx 